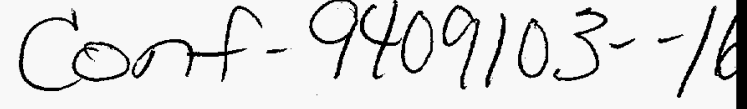

ANL-HEP-CP-94-62

For the Proceedings of the 11 th int. Symposium on High Energy Spin Physics and 8th Int. Symposium on Polarization Phenomena in Nuclear Physics Indiana Univ. . Bloomington, IN 15-22 Sep. 1994

\title{
A Possible Method to Produce a Polarized Antiproton Beam at Intermediate Energies
}

\author{
H. Spinka, E. W. Vaandering, ${ }^{a}$ and J.S. Hofmann ${ }^{b}$ \\ High Energy Physics Division \\ Argonne National Laboratory, Argonne, Mlinois 60439, USA
}

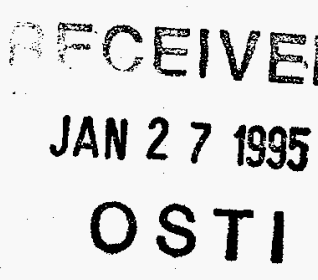

The lack of existence of polarized antiproton beams has severely limited the detailed understanding of the antinucleon-nucleon ( $\overline{\mathrm{N} N}$ ) interaction at low and intermediate energies. In particular, it would be desirable to have improved knowledge of the $\overline{\mathrm{N}} \mathrm{N}$ short range force and annihilation. A complete description of $\overline{\mathrm{N}} \mathrm{N} \rightarrow \overline{\mathrm{N}} \mathrm{N}$ scattering requires a determination of five complex scattering amplitudes for each of two isospins. At least 19 different spin observables must be measured at each c.m. angle and energy to reconstruct these amplitudes in a model independent fashion. However, only a few different types of spin observables for the $\bar{p} p \rightarrow \bar{p} p$ and $\bar{p} p \rightarrow \bar{n}$ reactions have been measured. This situation is similar to the status of the NN interactions in the early 1960's. Furthermore, the $\bar{N} N$ interaction is highly inelastic because of annihilation channels, even at the lowest beam moinenta, whereas NN scattering is essentially elastic up to about $800 \mathrm{MeV} / \mathrm{c}$. A polarized antiproton beam incident on a polarized proton or deuterum target would permit about a dozen new spin observables to be measured in $\overline{\mathrm{N} N} \rightarrow \overline{\mathrm{N}} \mathrm{N}$ scattering, as well as many new spin observables for $\overline{\mathrm{N}} \mathrm{N} \rightarrow \pi \pi_{\mathrm{K}} \mathrm{KK}$, etc. reactions, making an enormous impact on the understanding uit tiee $\overline{\mathrm{N}} \mathrm{N}$ interaction.

Several methods have been discussed to produce polarized $\overline{\mathrm{p}}$ beams at momenta up to a few $\mathrm{GeV} / \mathrm{c}[1-5]$. Three of these methods rely on dedicated use of the accelerator or a storage ring. The antiprotons are produced with a beam of high energy protons, collected, and finally stored in the accelerator or storage ring for hours while the $\bar{p}$ 's are slowly polarized. These methods involve formation of $\overline{\mathrm{H}}$ atoms [1,2], Stern-Gerlach separation of $\overline{\mathrm{p}}$ spins in an inhomogeneous magnetic field [3], and differences in $\bar{p} p$ total cross sections for parallel and antiparallel spins [4-6]. A fourth method would scatter an unpolarized $\bar{p}$ beam from carbon, but the resulting $\bar{p}$ beam polarization

a. Present address: University of Colorado, Boulder, CO 80309.

b. Present address: Visix Software, Inc., Reston, VA.

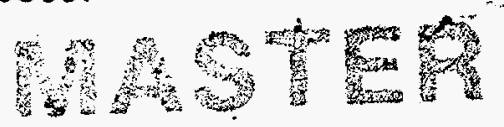




\section{DISCLAIMER}

This report was prepared as an account of work sponsored by an agency of the United States Government. Neither the United States Government nor any agency thereof, nor any of their employees, makes any warranty, express or implied, or assumes any legal liability or responsibility for the accuracy, completeness, or usefulness of any information, apparatus, product, or process disclosed, or represents that its use would not infringe privately owned rights. Reference herein to any specific commercial product, process, or service by trade name, trademark, manufacturer, or otherwise does not necessarily constitute or imply its endorsement, recommendation, or favoring by the United States Government or any agency thereof. The views and opinions of authors expressed herein do not necessarily state or reflect those of the United States Government or any agency thereof. 


\section{DISCLAIMER}

Portions of this document may be illegible in electronic image products. Images are produced from the best available original document. 
would be quite small [7]. This paper presents the conceptual design of a still different method, not requiring dedicated running in an accelerator such as LEAR. It would be ideal for a kaon factory beam line. Only one polarized antiproton beam has been successfully built [8], but its operation is based on different principles, and it operated at much higher energies $(\sim 200 \mathrm{GeV})$.

The method to produce a polarized $\bar{p}$ beam at intermediate energies is shown schematically in Fig. 1. A secondary beam of unpolarized antiprotons strikes a liquid hydrogen $\left(\mathrm{LH}_{2}\right)$ target. Elastically scattered $\overline{\mathrm{p}}$ 's are refocussed at the experimental target, for example a polarized proton target (PPT), with a toroidal magnet which accepts a range of scattering angles and azimuthal angles, $\phi$. The scattered $\overline{\mathrm{p}}$ 's have a known polarization [913] which is perpendicular to the scattering plane. If there is no bending magnet after the toroid, the $\overline{\mathrm{p}}$ 's will have transverse polarizations at the experimental target. Note that reversing the magnetic field direction would permit polarized protons to be collected and focussed on the target from an incident unpolarized proton beam. This would allow for tests of systematic errors with the well-studied pp elastic scattering reaction.

In the ideal case, no $\overline{\mathrm{p}}$ spin precession would occur for an incident beam with a small spot size, since the toroidal magnetic field would always be antiparallel to the $\overline{\mathrm{p}}$ spin direction. A realistic toroid design will limit the acceptance for antiprotons in $\phi$ due to the presence of conductor windings, and will cause small spin precessions due to a nonideal toroidal field. Small spin precessions will also occur when the ratio of the incident beam spot size to the toroid radius is sizeable.

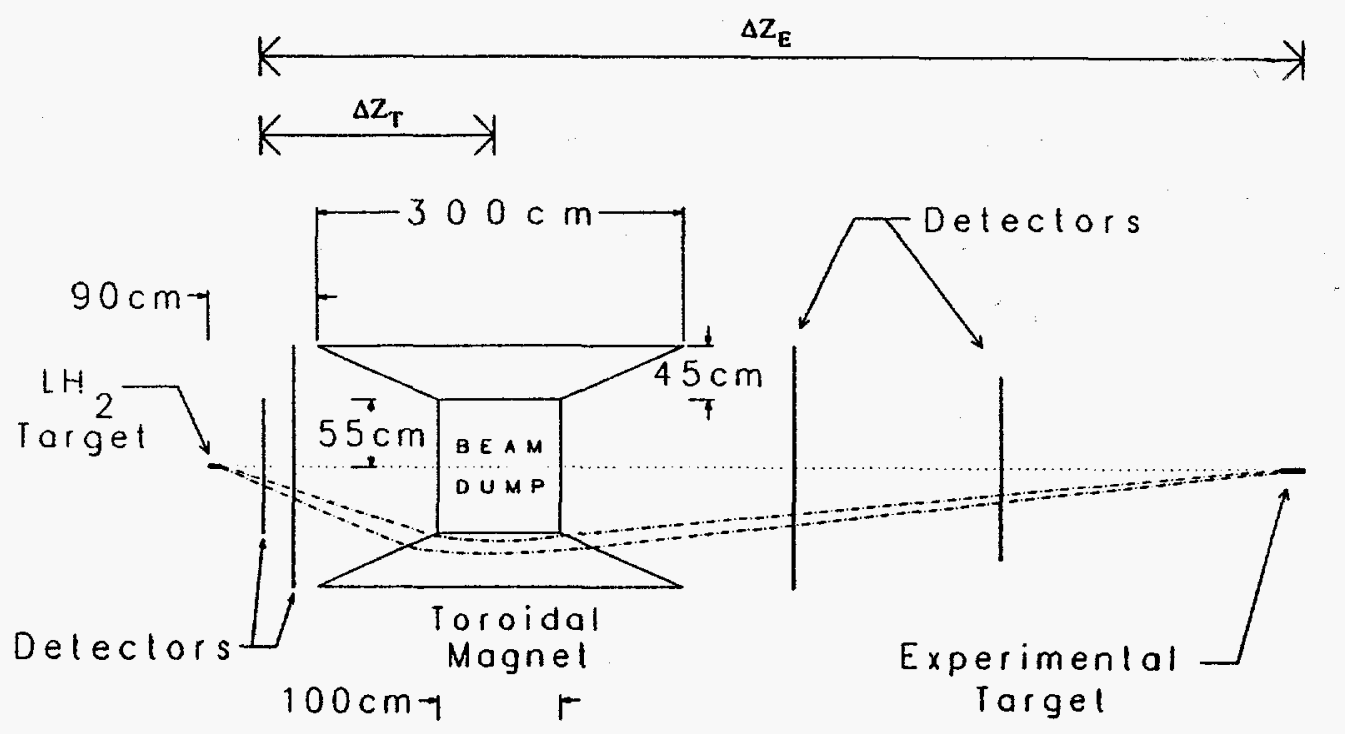

FIGURE 1. Drawing of a possible design for a polarized $\bar{p}$ beam line at $1089 \mathrm{MeV} / \mathrm{c}$. The incident unpolarized $\bar{p}$ 's enter from the left and strike the $\mathrm{LH}_{2}$ target. Two examples of scattered $\bar{p}$ 's are shown, along with detectors to measure the particle trajectories. 
There are a number of important considerations for the detailed design of (1) the toroid, (2) the choice of distance between the $\mathrm{LH}_{2}$ target and the toroid $\left(\Delta \mathrm{Z}_{\mathrm{T}}\right.$ - see Fig. 1), and (3) the distance from the $\mathrm{LH}_{2}$ target to the nominal beam focus at the experimental target $\left(\Delta Z_{E}\right)$. The distance $\Delta Z_{T}$ should be kept small in order to minimize the size and cost of the toroidal magnet. The beam line design should permit operation over a wide range of polarized beam momenta. The spot size at the focus should be minimized, since it is costly to construct a large diameter and volume polarized target. The ratio of distances $\Delta \mathrm{Z}_{\mathrm{E}} / \Delta \mathrm{Z}_{\mathrm{T}}$ should be large to reduce the beam divergence at the beam focus. The intensity of the polarized antiproton beam should be maximized to allow higher statistics experiments and/or to reduce running time per measurement. One way to achieve higher intensity is to design the beam line to accept sizeable divergence, momentum spread, and spot size for the unpolarized $\overline{\mathrm{p}}$ beam incident on the $\mathrm{LH}_{2}$ target. For example, a set of correction coils in addition to the main toroidal coils will allow a smaller focus for the polarized antiprotons at the experimental target over a wide range of momentum and other beam parameters. A realistic design will be a compromise of the considerations above and other factors, such as cost and available space.

The trajectories of the $\bar{p}$ 's scattered in the $\mathrm{LH}_{2}$ target must be measured with several sets of position sensitive detectors, such as multiwire proportional chambers. One reason is to verify that a $\bar{p}$ p elastic scattering occurred in the $\mathrm{LH}_{2}$ target, based on the observed scattering angle and particle bend in the toroid. A well separated $\bar{p}$ beam and/or a threshold Cerenkov to identify $\pi^{-}$and $\mathrm{K}^{-}$particles in the polarized beam will be highly desirable, since pions and kaons will scatter at forward angles in the $\mathrm{LH}_{2}$ target with kinematics similar to $\bar{p} p$ elastic scattering. Another reason to measure the trajectories of the scattered antiprotons is to tag the $\overline{\mathrm{p}}$ polarization direction and maznitude on a particle-by-particle basis, since these will vary with the beam phair space. Corrections to the antiproton spin direction due to a nonideal tcioidal field can also be included. A third reason is to measure the $\overline{\mathrm{p}}$ angle and position at the experimental target, so that the scattering angle and interaction point can be determined. This beam line design gives sizeable beam divergences, especially at low momenta.

Detailed calculations [14] were performed to obtain estimated intensities and polarizations for the polarized antiproton beam design of Fig. 1. A Monte Carlo computer program was written to perform these calculations. The incident unpolarized $\overline{\mathrm{p}}$ beam was assumed to be a secondary beam with $\pm 5 \%$ momentum spread, $\pm 5.0 \mathrm{mrad}$ divergence, and $\pm 1.0 \mathrm{~cm}$ spot size at the $\mathrm{LH}_{2}$ target. The target length was taken to be $10 \mathrm{~cm}$.

In the program, antiprotons were scattered in the $\mathrm{LH}_{2}$ target with a cross section and polarization calculated from Legendre polynomial fits [1113] to the experimental data from 0.4 to $1.7 \mathrm{GeV} / \mathrm{c}$ [9-13]. The square of 
the statistical uncertainty in spin observable measurements is proportional to $\mathrm{Q}^{-1}=\left(\mathrm{P}^{2} \mathrm{~d} \sigma / \mathrm{d} \Omega_{\text {c.m. }}\right)^{-1}$, where $\mathrm{P}$ and $\mathrm{d} \sigma / \mathrm{d} \Omega$ are the $\overline{\mathrm{p} p}$ elastic analyzing power and differential cross section. The quantity $Q$ can be used as a "figure of merit" to compare scattering at different angles, and it is desired to maximize Q. Fig. 2 shows typical data, fits, and estimates of $Q$ for one beam momentum as a function of 4-momentum transfer squared, $t$, from Ref. 11. The results of the fits suggest $Q$ is maximized for $-t \simeq 0.1-0.15(\mathrm{GeV} / \mathrm{c})^{2}$ for beam momenta above about $0.6 \mathrm{GeV} / \mathrm{c}$. The $\mathrm{LH}_{2}$ target to toroid distance $\Delta \mathrm{Z}_{\mathrm{T}}$ was chosen to give a central value of $\mathrm{t} \simeq-0.12(\mathrm{GeV} / \mathrm{c})^{2}$ accepted by the beam line for the Monte Carlo calculations.

FIGURE 2. Plots of the c.m. differential cross section, polarization, and figure of merit, $Q$, as functions of the 4-momentum transfer squared, $t$. The laboratory momentum is $1400 \mathrm{MeV} / \mathrm{c}$ and the data are from Ref. 11. The Legendre polynomial fits are shown as solid lines.

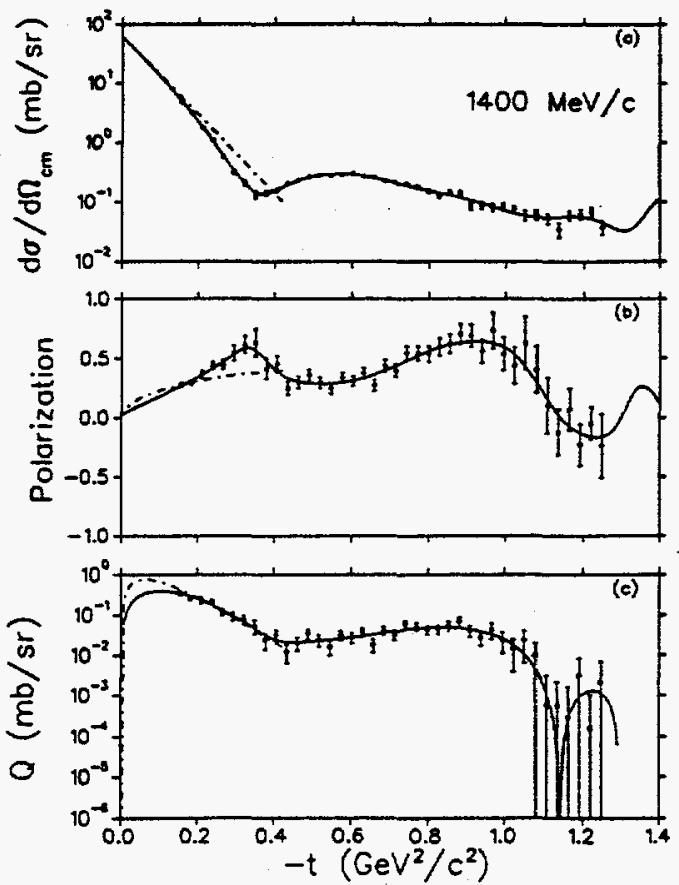

For simplicity, an idealized toroidal field was assumed. In cylindrical coordinates centered on the toroid, the magnitude of the magnetic field was assumed to vary as $\mathrm{r}^{-1}$. The magnetic field cross section was taken to be trapezoidal with values chosen to give the best beam line performance among a number of cases considered $(|z| \leq 0.5 \mathrm{~m}$ at $\mathrm{r}=0.55 \mathrm{~m}$ and $|\mathrm{z}| \leq 1.5 \mathrm{~m}$ at $\mathrm{r}=$ $1.0 \mathrm{~m}$ ). It was assumed that the toroid coils subtended half the $\phi$ acceptance at the $\mathrm{LH}_{2}$ target, and no correction coils were included. The maximum magnetic field was $1.3 \mathrm{~T}$ at $\mathrm{r}=0.55 \mathrm{~m}$.

The scattered antiproton trajectories were numerically integrated through the toroidal magnetic field in the Monte Carlo program. Particle trajectories were considered good if the $\overline{\mathrm{p}}$ 's passed through a 4-cm diameter, $10-\mathrm{cm}$ long cylinder representing the experimental target; other trajectories were rejected. Polarized targets with similar dimensions have been constructed and used in experiments. The value of $\Delta Z_{E}$ was varied in order to approximately maximize the fraction of good trajectories. 
The results of these calculations suggest that the $\bar{p}$ beam polarization and intensity will be typically 0.2 and $2 \times 10^{-4}$ per incident unpolarized antiproton, respectively, in the momentum range 0.5 to $2.5 \mathrm{GeV} / \mathrm{c}$. Several possibilities to increase the intensity exist, such as increasing the experimental target volume or the $\mathrm{LH}_{2}$ target length, or adding correction coils to the toroid. The intensity was also found to be sensitive to the incident unpolarized beam momentum spread, but nearly independent of divergence or spot size up to $\pm 20 \mathrm{mrad}$ and $\pm 1.5 \mathrm{~cm}$, respectively.

Reversal of polarization direction by $180^{\circ}$ is a common way to reduce systematic errors in spin experiments. For the transverse spins in the beam line in Fig. 1, this can be accomplished by adding a solenoid after the toroid to precess the spins by $\pm 90^{\circ}$. The change in solenoid current from $+90^{\circ}$ to $-90^{\circ}$ spin precession can occur in minutes, even for superconducting solenoids. With this option for reversal of the beam spin direction, experiments on unpolarized targets become feasible, such as for studies of polarized $\overline{\mathrm{p}}$ scattering from nuclear targets.

One problem is that the polarized $\overline{\mathbf{p}}$ beam in Fig. 1 contains no longitudinal polarization. This can be solved by the addition of three dipole magnets as in Fig. 3 and Ref. 15. This solution keeps the average beam position and direction fixed at the experimental target, so that the detectors after the beam focus would not need to move as a function of beam momentum. The bend in the final dipole, $\theta=90^{\circ} \cdot m /[E \cdot(g / 2-1)]$, is momentum dependent and is determined by the requirement that the $\overline{\mathrm{p}}$ spin precess by $90^{\circ}$ in the horizontal plane. The $\overline{\mathrm{p}}$ mass and energy in the expression above are $\mathrm{m}$ and $\mathrm{E}$. Knowledge of the field map of the final dipole magnet and of the $\overline{\mathrm{p}}$ trajectory will enable the spin precession to be accurately computed for each particle, even for cases with large beam divergence at low momenta.

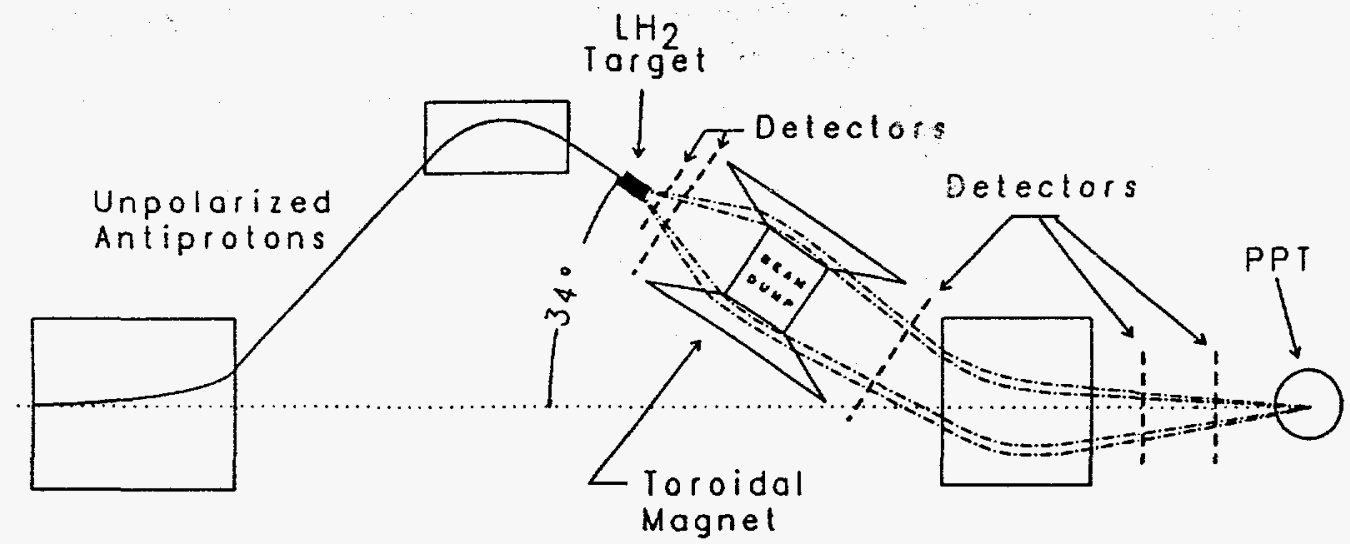

FIGURE 3. Drawing of a possible design for a $\sim 1.0 \mathrm{GeV} / \mathrm{c}$ transversely and longitudinally polarized $\bar{p}$ beam line. The bending magnets are required to keep the average angle of the polarized antiprotons at the experimental target fixed as a function of momentum. 
A feasible and conservative design for a medium energy polarized antiproton beam has been presented. The design requires an intense beam of unpolarized antiprotons $\left(\geq 10^{7} / \mathrm{sec}\right)$ from a typical secondary beam line in order to achieve reasonable $\bar{p}$ p elastic scattering count rates. All three beam spin directions can be achieved. Methods were discussed to reverse the spin directions in modest times, and to change to a polarized proton beam if desired. It is expected that experiments with such a beam would have a profound effect on the understanding of the $\overline{\mathrm{N}} \mathrm{N}$ interaction at intermediate energies.

We wish to express our gratitude to our colleagues at Valparaiso University, CEA Saclay, France, and Argonne National Laboratory for helpful suggestions during the course of this work. We are also thankful for help with some of the figures by $\mathrm{D}$. Lopiano. This work was supported in part by the U.S. Department of Energy, Division of High Energy Physics, Contract W-31-109-ENG-38.

\section{References}

1. K. Imai, Proc. 6th Int. Symp. on Polarization Phenomena in Nuclear Physics, Osaka, Japan, Eds. M. Kondo et al., Suppl. Jour. Roy. Soc. Japan 55, 11361139 (1986).

2. H. Poth, Proc. Conf. on Intersections Between Particle and Nuclear Physics, Lake Louise, Canada, Amer. Inst. Phys. Conf. Proc. 150, 480-489 (1986).

3. Y. Onel, A. Penzo, and R. Rossmanith, Proc. Conf. on Intersections Between Particle and Nuclear Physics, Lake Louise, Canada, Amer. Inst. Phys. Conf. Proc. 150, 1229-1231 (1986); T.O. Ninikoski and R. Rossmanith, Nucl. Instr. Meth. A255, 460-465 (1987).

4. P.L. Csonka, Nucl. Instr. Meth. 63, 247-252 (1968).

5. G. Graw, Physics With Polarized Beams on Polarized Targets, ods. J. Sowinski and S.E. Vigdor (World Scientific, Singapore, 1990) pp. $20 \hat{2}-348$.

6. F. Rathmann et al., Phys. Rev. Lett. 71, 1379-1382 (1993).

7. R. Birsa et al., Phys. Lett. 155B, 437-441 (1985).

8. D.P. Grosnick et al., Nucl. Instr. Meth. A290, 269-292 (1990).

9. C. Daum et al., Nucl. Phys. B6, 617-627 (1968).

10. M.G. Albrow et al., Nucl. Phys. B37, 349-363 (1972).

11. R.A. Kunne et al., Nucl. Phys. B323, 1-36 (1989).

12. R. Bertini et al., Phys. Lett. 228B, 531-535 (1989).

13. F. Perrot-Kunne et al., Phys. Lett. 261B, 188-190 (1991).

14. E.W. Vaandering, H.M. Spinka, and J.S. Hofmann, accepted for publ. in Nucl. Instr. Meth. A.

15. E. Colton et al., Nucl. Instr. Meth. 151, 85-88 (1978); E.P. Colton, IEEE Trans. Nucl. Sci. NS-26, 3206-3208 (1979); H. Spinka et al., Nucl. Instr. Meth. 211, 239-261 (1983). 